\title{
ÉTABLISSEMENT DE COURBES DE PRÉFÉRENCES D'HABITAT POUR LES FRAYĖRES ET LES JEUNES STADES D'OMBRE COMMUN (THYMALLUS THYMALLUS, L.).
}

\author{
P. SEMPESKI, P. GAUDIN \\ URA CNRS 1974, Ecologie des Eaux Douces et des Grands Fleuves, \\ 43 bd du 11 novembre 1918, 69622 Villeurbanne Cedex, France.
}

\begin{abstract}
RÉSUMÉ
Des courbes de préférences d'habitat ont été construites pour les frayères et les jeunes stades d'ombre commun pour les paramètres suivants : vitesse du courant, profondeur et granulométrie du substrat. La pertinence de ces courbes, construites par stade de développement, est discutée à partir de la notion de "groupe fonctionnel".

\section{CONSTRUCTION OF HABITAT PREFERENCE CURVES FOR SPAWNING SITES AND YOUNG STAGES OF GRAYLING (THYMALLUS THYMALLUS, L.).}

\begin{abstract}
Habitat preference curves were built for spawning sites and young stages of grayling for the following parameters : water velocity, depth and substrate. The pertinence of such curves, built for each developmental stage, is discussed from the notion of "functional group".
\end{abstract}

\section{INTRODUCTION}

Les modèles courants de prédiction de la capacité d'accueil d'un cours d'eau ou d'un segment de cours d'eau, pour une espèce de poisson et un stade de développement donnés, sont basés sur le couplage de modèles physiques et biologiques quantifiant respectivement l'habitat disponible et les préférences d'habitat des différents stades (BOVEE, 1982 ; SOUCHON et al., 1989). Dans le cadre de la méthode des microhabitats, les préférences d'habitat sont exprimées sous forme de courbes, pour trois paramètres classiques : la vitesse du courant, la profondeur et la granulométrie du substrat. Si les Salmonidés comme la truite et le saumon ont bénéficié d'efforts de recherche considérables dans ce domaine ces dernières années (voir par exemple RIMMER et al., 1984 ; DEGRAAF et BAIN, 1986 ; MORANTZ et al., 1987 ; HEGGENES, 1991), tel n'a pas été le cas de leur proche parent, l'Ombre commun (Thymallus thymallus), pour lequel seules des courbes d'experts sont disponibles (BULLOCK et al., 1991). Pourtant, cette espèce a un intérêt économique non négligeable, notamment par l'engouement qu'elle suscite auprès des pêcheurs sportifs. De plus, les populations d'ombre sont en voie de régression en Europe et en particulier en France (PERSAT, 1976, 1988), du fait principalement des aménagements et de la régulation des cours d'eau qu'ils habitent. II est donc nécessaire d'étudier avec précision les relations existant entre ce poisson et son habitat, en particulier au niveau des jeunes stades, qui sont reconnus comme une période sensible du cycle (ELLIOTT, 1989).

Le but de cette étude a été de construire un jeu de courbes de préférences d'habitat pour les frayères et les jeunes stades d'ombre, mais également de discuter de la pertinence de telles courbes, à la lumière de résultats récents concernant la dynamique de l'utilisation de l'habitat par les jeunes stades (SEMPESKI et GAUDIN, sous presse). 


\section{MATÉRIEL ET MÉTHODES}

\section{Etablissement de courbes de préférence}

Les données ont été récoltées sur le Pollon, petit affluent phréatique de la rivière d'Ain, d'un débit moyen annuel de $1.1 \mathrm{~m}^{3 / \mathrm{s}}$ et d'une largeur moyenne d'environ $8.5 \mathrm{~m}$ au niveau du site d'étude. Les caractéristiques physiques de 83 frayères ont été décrites en mars 1992, selon un protocole détaillé dans SEMPESKI et GAUDIN (1995a). Les habitats des alevins (longueur totale $=15-30 \mathrm{~mm}$ ) et de juvéniles de 100 à $150 \mathrm{~mm}$ ont été repérés, délimités, puis décrits à l'aide d'un échantillonnage aléatoire respectivement en septembre et novembre 1993 (SEMPESKI et GAUDIN 1995b). Etant donnée la grande stabilité hydrologique du Pollon, l'habitat disponible n'a été décrit qu'une fois en juillet 1992, au niveau d'un segment de $300 \mathrm{~m}$ de long comprenant trois séquences mouilleradier et incluant l'ensemble des habitats utilisés pour la reproduction et par les jeunes stades, selon un protocole inspiré de STATZNER et MÜLLER (1989) détaillé dans SEMPESKI et GAUDIN (1995a).

Les différents types d'habitat ont été décrits à partir de paramètres classiques. La vitesse du courant a été mesurée à 0.4 fois la profondeur (vitesse moyenne) et à $5 \mathrm{~cm}$ audessus du fond (vitesse au fond) à l'aide d'un micromoulinet à hélice. La profondeur a été mesurée au centimètre près avec une règle graduée. La granulométrie du substrat a été estimée visuellement en classes, selon un code et une échelle tirée de SOUCHON et al. (1989) : limon, L, (3.9-62.5 $\mu \mathrm{m})$, sable fin, SF, $(62.5-500 \mu \mathrm{m})$, sable grossier, $\mathrm{SG},(500 \mu \mathrm{m}-$ $2 \mathrm{~mm})$, graviers fins, GF, (2-8 mm), graviers grossiers, GG, $(8-16 \mathrm{~mm})$, cailloux fins, CF, (16-32 mm), cailloux grossiers, CG, $(32-64 \mathrm{~mm})$, pierres fines, $P F,(64-128 \mathrm{~mm})$, pierres grossières, PG, (128-256 mm), blocs, $B,(256-1024 \mathrm{~mm})$, rocher, dalle, $R,(>1024 \mathrm{~mm})$.

Un coefficient de préférence $\mathrm{C}(\mathrm{x}, \mathrm{i})$ a été calculé, pour chaque modalité i de chaque variable $x$, selon la formule suivante :

$$
\mathrm{C}(\mathrm{x}, \mathrm{i})=(\mathrm{Ni} / \mathrm{Nt}) /(\mathrm{Fi} / \mathrm{Ft})
$$

avec $\mathrm{Ni} / \mathrm{Nt}$ : nombre de poissons associés à la modalité i/nombre total de poissons,

$\mathrm{Fi} / \mathrm{Ft}$ : nombre de points associés à la modalité i/nombre total de points échantillonnés.

$C(x, i)$ est ensuite exprimé comme une proportion du coefficient de préférence maximum pour la variable $x$.

\section{Utilisation jour/nuit de I'habitat}

Des suivis par observations directes depuis la berge, réalisés entre avril et juillet 1993 sur cette même rivière, ont permis de caractériser les habitats utilisés de jour et de nuit par les jeunes stades (SEMPESKI et GAUDIN, sous presse). Les repérages ont été effectués en remontant la rivière, depuis la berge ou dans le chenal, en utilisant pour les relevés nocturnes une lampe torche à la manière d'un flash. Pour chaque individu ont été estimés : la longueur totale du corps, la position relative par rapport à la berge et à la limite du chenal, la hauteur d'eau sélectionnée, la position dans la colonne d'eau. Au total, 723 individus ont été observés de jour et 507 de nuit. Ce travail a permis de mettre en évidence une organisation en trois "groupes fonctionnels" : le groupe des alevins post-émergents de 15 à $20 \mathrm{~mm}$, localisés en marge des zones mortes très près de la berge, le groupe des alevins âgés et jeunes juvéniles regroupant les individus de 20 à $40 \mathrm{~mm}$. encore inféodés à la zone morte mais tendant à se rapprocher de la limite du courant et du fond, et le groupe des juvéniles $>40 \mathrm{~mm}$ observés dans le chenal en position benthique. Nous présenterons ici uniquement les résultats concernant l'évolution de la hauteur d'eau sélectionnée par les jeunes stades d'ombre de jour et de nuit.

\section{RÉSULTATS}

\section{Courbes de préférences d'habitat par stade de développement}

Les courbes de préférence d'habitat construites pour les frayères, les alevins et les juvéniles indiquent des exigences très strictes pour chacun des différents stades étudiés vis-à-vis des paramètres physiques (Fig. 1). Les géniteurs d'ombre sélectionnent leurs sites 
de ponte de préférence en des points caractérisés par une vitesse moyenne de courant essentiellement située dans la gamme $40-60 \mathrm{~cm} \mathrm{~s}^{-1}$, une hauteur d'eau assez faible $(20-30 \mathrm{~cm})$ et un substrat composé de graviers grossiers et cailloux fins $(8-32 \mathrm{~mm})$. Les alevins ont une préférence pour des vitesses de courant très faibles $\left(<20 \mathrm{~cm} \mathrm{~s}^{-1}\right)$, des profondeurs également faibles $(<20 \mathrm{~cm})$, et un substrat constitué de limon et sable fin $(<500 \mu \mathrm{m})$. Les juvéniles d'automne, quant à eux, sélectionnent des zones du chenal moyennement courantes $\left(20-60 \mathrm{~cm} \mathrm{~s}^{-1}\right)$, moyennement profondes $(40-60 \mathrm{~cm})$, sur un substrat de granulométrie moyenne du même type que les frayères.
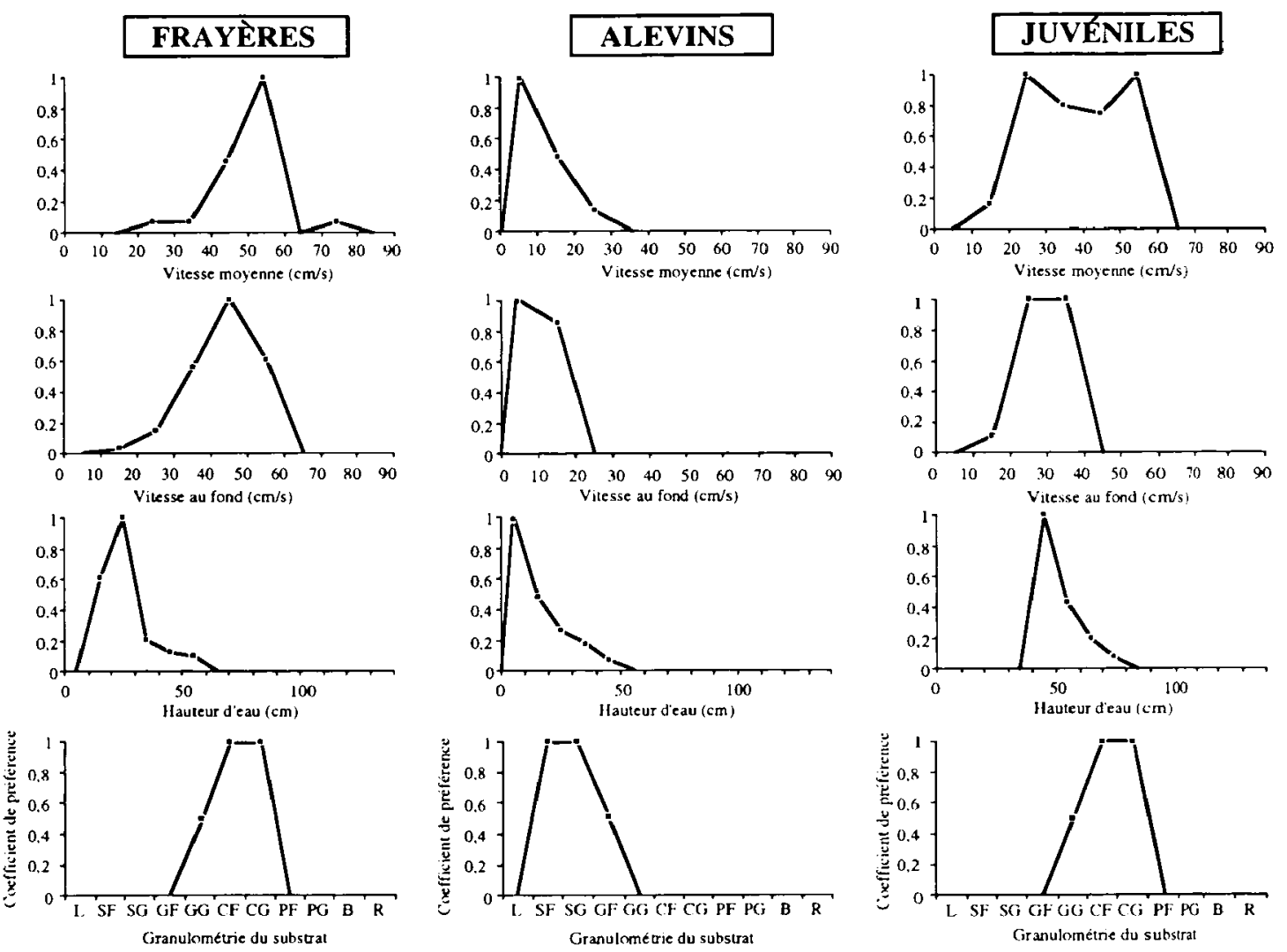

Figure 1 : Courbes de préférences d'habitat construites pour les frayères à ombre (83 frayères décrites) et les stades alevin (77 points décrits) et juvénile (24 points décrits) (d'après SEMPESKI et GAUDIN, 1995a et b).

Figure 1 : Habitat suitability curves built for grayling spawning sites $(n=83)$, and larval (77 spots described) and juvenile (24 spots described) stages (from SEMPESKI and GAUDIN, 1995a and b).

\section{Courbes d'utilisation de l'habitat par "groupe fonctionnel" : exemple de la profondeur}

L'analyse de la hauteur d'eau sélectionnée par l'ensemble des ombrets, toutes classes de taille confondues, montre une différence nette entre le jour et la nuit (Fig. 2A). Ainsi, les ombrets occupent de jour des profondeurs principalement situées entre 10 et $40 \mathrm{~cm}$, tandis que de nuit on les observe dans des profondeurs plus faibles, essentiellement $<10 \mathrm{~cm}$.

La réorganisation des données en groupes fonctionnels montre que les distributions observées pour chacun des trois groupes sont significativement différentes de jour comme de nuit (Chi-2, $N=300, p<0.001$ dans les deux cas, Fig. 2B). Le groupe des postémergents de 15-20 mm exploite de jour des profondeurs essentiellement comprises entre 10 et $30 \mathrm{~cm}$, alors que de nuit ils occupent des hauteurs d'eau plus faibles, plus de $80 \%$ d'entre eux étant observés dans moins de $10 \mathrm{~cm}$ d'eau, dont $60 \%$ dans moins de $5 \mathrm{~cm}$. Si 
A. Représentation toutes classes de tailles confondues

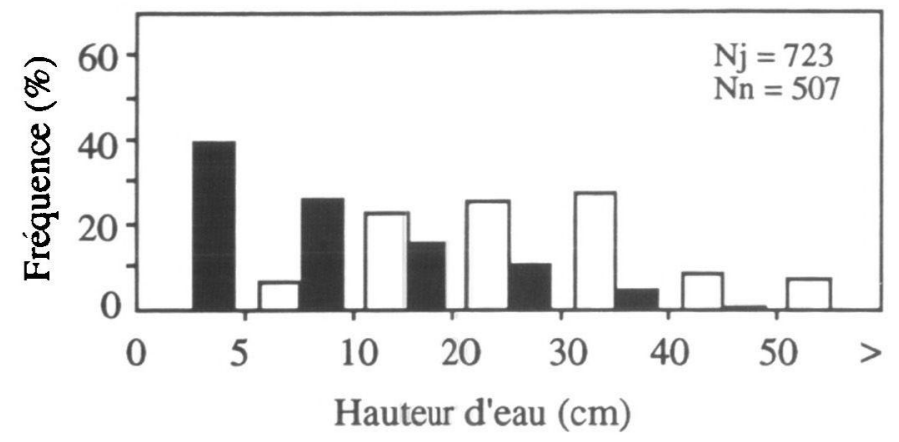

JOUR

NUIT

B. Représentation par groupe fonctionnel

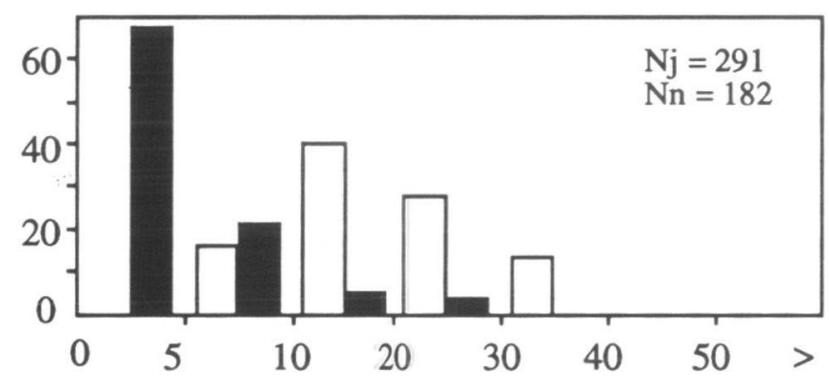

Groupe 1

$15-20 \mathrm{~mm}$

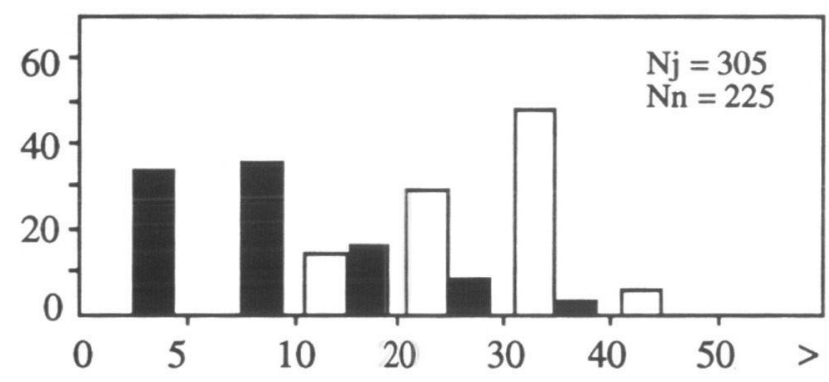

Groupe 2

$20-40 \mathrm{~mm}$

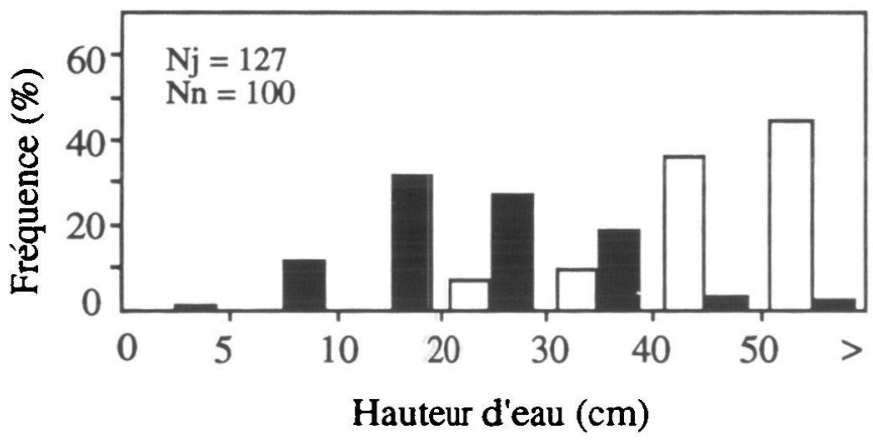

Groupe 3

$>40 \mathrm{~mm}$

Figure 2 : Histogrammes jour/nuit d'utilisation de la profondeur par les jeunes ombres, toutes classes de tailles confondues (A) ou par groupe fonctionnel (B). $\mathrm{Nj}$ et $\mathrm{Nn}$ représentent les nombres d'individus observés, respectivement de jour et de nuit.

Figure 2 : Day/night water depth use histogramms for young grayling, all size classes pooled (A) or by functional group (B). $\mathrm{Nj}$ and $\mathrm{Nn}$ represent the numbers of individuals observed by day and by night, respectively. 
la distribution nocturne des $20-40 \mathrm{~mm}$ est proche de celle des $15-20 \mathrm{~mm}$, de jour $80 \%$ des représentants de ce deuxième groupe s'observent dans la gamme de profondeurs $20-40 \mathrm{~cm}$. Au-delà de $40 \mathrm{~mm}$, les ombrets sont essentiellement rencontrés de jour dans des profondeurs supérieures à $40 \mathrm{~cm}$, et de nuit principalement dans 10 à $40 \mathrm{~cm}$ d'eau, les très faibles profondeurs $(<5 \mathrm{~cm})$ étant alors délaissées.

\section{DISCUSSION}

Les courbes de préférence d'habitat présentées ici doivent être considérées comme un jeu de courbes préliminaires, dans la mesure où elles ont été bâties à partir de données provenant d'une seule rivière. Néanmoins, les résultats sont en accord avec les descriptions données dans la littérature pour les frayères (FABRICIUS et GUSTAFSON, 1955 ; MÜLLER, 1961 ; GÖNCZI, 1989), comme pour les jeunes stades (MÜLLER, 1961 ; SCOTT, 1985 ; BARDONNET et al., 1991). Si les courbes concernant la vitesse du courant confirment les courbes établies par les experts anglais (BULLOCK et al., 1991), et ce quel que soit le stade considéré, des différences assez nettes s'observent au niveau de la profondeur et la granulométrie du substrat. Dans le cas de la profondeur, les gammes de valeurs proposées par les experts sont plus larges que les nôtres (30-120 cm pour les frayères, $12-36 \mathrm{~cm}$ pour les alevins et $60-200 \mathrm{~cm}$ pour les juvéniles). II est difficile de discuter ces différences, dans la mesure où les gammes de tailles des poissons correspondant à chacun des stades étudiés ne sont pas précisées, de même que manquent des informations sur l'habitat disponible. Or, il est reconnu que la sélection de l'habitat par une espèce et un stade donnés va dépendre de la disponibilité en habitat du cours d'eau considéré et également de la présence de compétiteurs et de prédateurs potentiels (GREENBERG, 1992).

La validité des courbes présentées ici, construites de manière classique par stade de développement, peut être discutée car elles ont été élaborées à partir de données récoltées de jour, et ne donnent donc qu'une image partielle de l'habitat préférentiellement utilisé. Ceci est d'autant plus ennuyeux que l'habitat de nuit est souvent plus limitant que l'habitat de jour (HARRIS et al., 1992). De plus, le découpage de ces courbes par stade de développement ne correspond pas forcément à une réalité biologique, comme le suggèrent les résultats concernant la dynamique spatio-temporelle d'utilisation de l'habitat (ici la profondeur), organisée en groupes fonctionnels. Cette notion de "groupe fonctionnel" nous paraît fondamentale à développer dans le cadre de l'élaboration de courbes de préférence d'habitat, car elle est de toute évidence plus pertinente que celle de stade de développement. En particulier, elle a permis de déceler certaines phases plus sensibles que d'autres (par exemple, la nuit pour le groupe des $15-20 \mathrm{~mm}$ ), susceptibles de constituer des goulots d'étranglements pour la population et donc de représenter des phases "clés" dans la dynamique de la population (ELLIOTT, 1989). II serait donc utile, afin d'augmenter la précision et la pertinence des courbes de préférence d'habitat, de reconsidérer leur découpage en stades de développement en prenant en compte, par une analyse plus fonctionnelle, la véritable dynamique spatio-temporelle d'utilisation de l'habitat par une espèce.

\section{REMERCIEMENTS}

Nous voudrions remercier O. GROLET, C. HENRY, M. PHILIPPE, P. ROGER, $P$. SAGNES et $S$. VALENTIN pour leur aide sur le terrain. Ces travaux ont été effectués dans le cadre d'un Programme Interdisciplinaire de Recherche Environnement du CNRS (P.I.R.E.) concernant "les relations entre le poisson et son habitat".

\section{BIBLIOGRAPHIE}

BARDONNET A., GAUDIN P., PERSAT H., 1991. Microhabitats and diel downstream migration of young grayling (Thymallus thymallus L.). Freshwat. Biol., 26, 365-376.

BOVEE K.D., 1982. A guide to stream habitat analysis using the Instream Flow Incremental Methodology. Instream Flow Information Paper 12, U.S. Fish and Wildlife Service FWS/OBS 82/26, $248 p$. 
BULLOCK A., GUSTARD A., GRAINGER E. S., 1991. Instream flow requirements of aquatic ecology in two British rivers. Inst. Hydrol., Report no. 115, 138 p.

DE GRAAF D.A., BAIN L.H., 1986. Habitat use by and preferences of juvenile Atlantic salmon in two Newfoundland rivers. Trans. Am. Fish. Soc., 115, 671-681.

ELLIOTT J.M., 1989. Mechanisms responsible for population regulation in young migratory trout, Salmo trutta. I. The critical time for survival. J. Anim. Ecol., 58, 987-1001.

FABRICIUS E., GUSTAFSON K.J., 1955. Observations on the spawning behaviour of the grayling, Thymallus thymallus (L.). Rep. Inst. Freshwat. Res. Drottningholm, 36, 75-103.

GÖNCZI A.P., 1989. A study of physical parameters at the spawning sites of the European grayling (Thymallus thymallus L.). Reg. Rivers Res. Manage., 3, 221-224.

GREENBERG L.A., 1992. The effect of discharge and predation on habitat use by wild and hatchery brown trout (Salmo trutta). Reg. Riv. Res. Manage., 7, 205-212.

HARRIS D.D., HUBERT W.A., WESCHE T.A., 1992. Habitat use by young-of-year brown trout and effects on weighted usable area. Rivers, 3, 99-105.

HEGGENES J., 1991. Comparisons of habitat availability and habitat use by an allopatric cohort of juvenile Atlantic salmon Salmo salar under conditions of low competition in a Norwegian stream. Holarctic Ecol., 14, 51-62.

MORANTZ D.L., SWEENEY R.K., SHIRVELL C.S., LONGARD D.A., 1987. Selection of microhabitat in summer by juvenile Atlantic salmon (Salmo salar). Can. J. Fish. Aquat. Sci., 44, 120-129.

MÜLLER K., 1961. Die Biologie der Äsche (Thymallus thymallus L.) im Lule Älv (Schwedisch Lappland). Zeit. Fisch. Hilfswissenschaften, 1-3, 173-201.

PERSAT H., 1976. Principaux aspects de l'écologie de l'Ombre commun Thymallus thymallus (L. 1758) (Poissons Salmonidés). Thèse 3ème cycle, Univ. Lyon 1, 69 p.

PERSAT H., 1988. De la biologie des populations de l'ombre commun Thymallus thymallus (L. 1758) à la dynamique des communautés dans un hydrosystème fluvial aménagé, le Haut-Rhône français. Eléments pour un changement d'échelles. Thèse d'état, Univ. Lyon 1, $223 \mathrm{p}$.

RIMMER D.M., PAIN U., SAUNDERS R.L., 1984. Changes in the selection of microhabitat by juvenile Atlantic salmon (Salmo salar) at the summer-autumn transition in a small river. Can. J. Fish. Aquat. Sci., 41, 469-475.

SCOTT A., 1985. Distribution, growth, and feeding of postemergent grayling Thymallus thymallus in an English river. Trans. Am. Fish. Soc., 114, 525-531.

SEMPESKI P., GAUDIN P., 1995a. Habitat selection by grayling. I. Spawning habitats. J. Fish Biol., 47, 256-265.

SEMPESKI P., GAUDIN P., 1995b. Habitat selection by grayling. II. Preliminary results on larval and juvenile daytime habitats. J. Fish Biol., 47, 345-349.

SEMPESKI P., GAUDIN P., 1995. Size-related changes in diel distribution of young grayling (Thymallus thymallus). Can. J. Fish. Aquat. Sci. (sous presse).

SOUCHON Y., TROCHERIE F., FRAGNOUD E., LACOMBE C., 1989. Les modèles numériques des microhabitats des poissons : application et nouveaux développements. Rev. Sci. Eau, 2, 807-830.

STATZNER B., MÜLLER R., 1989. Standard hemispheres as indicators of flow characteristics in lotic benthos research. Freshwat. Biol., 21, 445-459. 